\title{
Role of plastic deformation in tailoring ultrafine microstructure in nanotwinned diamond for enhanced hardness
}

\author{
Wentao $\mathrm{Hu}^{1 \dagger}$, Bin Wen ${ }^{1 \dagger}$, Quan Huang ${ }^{2 \dagger}$, Jianwei Xiao ${ }^{1}$, Dongli $\mathrm{Yu}^{1}$, Yanbin Wang ${ }^{3}$, Zhisheng Zhao ${ }^{1}$, \\ Julong $\mathrm{He}^{1}$, Zhongyuan $\mathrm{Liu}^{1}, \mathrm{Bo} \mathrm{Xu}^{1}$ and Yongjun Tian ${ }^{1 *}$
}

\begin{abstract}
Nanotwinned diamond (nt-diamond), which demonstrates unprecedented hardness and stability, is synthesized through the martensitic transformation of onion carbons at high pressure and high temperature (HPHT). Its hardness and stability increase with decreasing twin thickness at the nanoscale. However, the formation mechanism of nanotwinning substructures within diamond nanograins is not well established. Here, we characterize the nanotwins in nt-diamonds synthesized under different HPHT conditions. Our observation shows that the nanotwin thickness reaches a minimum at $\sim 20 \mathrm{GPa}$, below which phase-transformation twins and deformation twins coexist. Then, we use the density-functional-based tight-binding method and kinetic dislocation theory to investigate the subsequent plastic deformation mechanism in these pre-existing phase-transformation diamond twins. Our results suggest that pressure-dependent conversion of the plastic deformation mechanism occurs at a critical synthetic pressure for nt-diamond, which explains the existence of the minimum twin thickness. Our findings provide guidance on optimizing the synthetic conditions for fabricating nt-diamond with higher hardness and stability.
\end{abstract}

Keywords: nanotwinned diamond, high temperature and high pressure (HTHP), plastic deformation; hardness

\section{INTRODUCTION}

Diamond, the stiffest and hardest material, has a wide range of industrial applications. In past decades, numerous efforts have been made to enhance its hardness [1-4], which has been shown to increase as the grain size and/or twin thickness is reduced to the nanometer scale according to the well-known Hall-Petch effect [5-7]. Nanograined diamonds (ng-diamonds) with grain sizes of 10-30 nm exhibit a Knoop hardness of 110-140 GPa [4], compared to a single-crystal hardness of up to $\sim 120 \mathrm{GPa}$. Further decreasing the grain size remains a great technical challenge owing to the high excess energy of grain boundaries and the very high synthesis temperature, which promote grain growth.

Nanotwinning provides a more effective approach to further refining the microstructure than nanograining does because twin boundaries possess much lower excess energy [8]. Through the transformation of onion-structured precursors at high pressure and high temperature (HPHT), nanotwinned cubic boron nitride and nanotwinned diamond (nt-diamond) were successfully synthesized $[9,10]$ and showed dramatically enhanced hardness, fracture toughness, and thermal stability compared to the coarse-grained counterparts. In the recovered nt-diamond samples, ultrafine $\{111\}$ nanotwins with an average twin thickness $(\lambda)$ of $5-8 \mathrm{~nm}$ were formed within the nanograins, resulting in an unprecedented Vickers hardness $\left(H_{\mathrm{V}}\right)$ of 175-200 GPa. Experimental results and theoretical analyses [9-14] indicate that both the Hall-Petch effect and quantum confinement effect contribute to the enhancement of the hardness, so that hardening continues as $\lambda$ decreases to $\sim 5 \mathrm{~nm}$ [14-16]. Is it possible to further reduce $\lambda$ to obtain nt-diamond with an even higher hardness? To answer this question, a deeper understanding of the mechanism of nanotwinning is necessary.

The face-centered cubic (fcc) diamond structure consists

${ }^{1}$ State Key Laboratory of Metastable Materials Science and Technology, Yanshan University, Qinhuangdao 066004, China

${ }^{2}$ Center for High Pressure Science \& Technology Advanced Research, Shanghai 201203, China

${ }^{3}$ Center for Advanced Radiation Sources, University of Chicago, Chicago, IL 60439, USA

† These authors contributed equally to this work.

*Corresponding author (email: fhcl@ysu.edu.cn) 
of two interpenetrating fcc sublattices shifted relative to one another along the body diagonal by $[1 / 4,1 / 4,1 / 4]$ of the unit cell. The fcc lattice can be visualized by considering three identical sheets of atoms stacked in the $\{111\}$ plane with a relative displacement of $1 / 2\langle 110\rangle$. Considering the two sublattices in diamond, the stacking sequence can be described as ...AaBbCc..., where A, B, C and a, b, c represent the three stacking planes of the two sublattices, respectively (Fig. S1, Supporting information (SI)). Therefore, the diamond $\{111\}$ planes have the highest lattice site density with the shortest lattice translations $(1 / 2\langle 110\rangle)$ and are the preferred slip planes. The main slip system is therefore of the $\{111\}\langle 110\rangle$ type. Plastic deformation of diamond occurs under very high shear stresses, which can be achieved either by indentation $[17,18]$ or by subjecting diamond crystals to an environment with a large pressure gradient, for example, by loading of diamond anvils in high-pressure diamond-anvil cell experiments [19]. As in other diamond-structured cubic materials such as $\mathrm{Si}$ and Ge [20-25], slip on $\{111\}$ can occur by dislocations either within the densely populated compound layers (e.g., $\mathrm{aB}$, $\mathrm{bC}$, or $\mathrm{cA}$ ) or between adjacent compound layers (e.g., Aa, $\mathrm{Bb}$, or $\mathrm{Cc}$ ). Dislocations that facilitate the two slips are known as glide-set and shuffle-set dislocations, respectively (Fig. S1). Generally, the glide-set dislocations are energetically favored, as they involve shorter Burgers vectors. Dissociated glide-set dislocations can induce stacking faults that cause deformation twins via slipping of Shockley partial dislocations in the $\{111\}$ planes [26,27]. Shuffle-set dislocations, on the other hand, are non-dissociated perfect dislocations and cannot create stacking faults and twins $[26,28]$.

Twins are common in natural and synthetic diamonds as a type of planar defect [29-32], which can be produced via both phase transformation [33-36] and deformation [26,37-39]. Both ng- and nt-diamond can be obtained from different carbon precursors through HPHT syntheses without resorting to catalysts $[4,10]$. For ng-diamond, some transformation or growth twins with relatively large $\lambda$ were observed in nanograins originating in the graphite-diamond transformation process [35]. Ubiquitous nanotwins within nanograins were observed in nt-diamond samples that were transformed from onion carbon precursors at $18-25 \mathrm{GPa}$ and $1850-2000^{\circ} \mathrm{C}$ [10]. These nanotwins also originate from either transformation or growth twinning. In our previous study [10], however, we noticed that a maximal $H_{\mathrm{V}}$ of $\sim 203 \mathrm{GPa}$ occurs at a synthesis pressure of $\sim 20 \mathrm{GPa}$, which corresponds to a minimum in $\lambda$. The correlation between $H_{\mathrm{V}}$ and $\lambda$ cannot be fully understood in terms of the transformation of the onion carbon because this correlation should be monotonic if $\lambda$ was related to the nucleation or growth of the diamond phase alone. The turnover of $\lambda$ (and $H_{\mathrm{V}}$ ) with pressure implies that twinning with other origins may coexist with transformation or growth twinning during HPHT synthesis of nt-diamond.

Previous experimental results revealed coexisting dislocations and deformation twins in sintered micron-sized diamond powders [36]. At 7.7 GPa, numerous twins and dense stacking faults were observed in diamond recovered from temperatures of $700-1400^{\circ} \mathrm{C}$, but only a few twins were observed in samples treated above $1400^{\circ} \mathrm{C}$ [36]. $\{111\}$ Twin planes are the only possible coherent boundaries for deformation twinning in diamond [40]. \{111\} Deformation twins can be produced by shearing the diamond

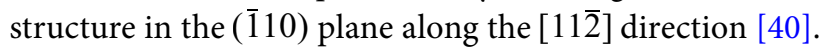
However, experimental data on deformation twinning as a function of pressure and temperature are scarce owing to the well-known technical difficulties in deforming diamond. In this study, we characterize nanotwins in nt-diamond samples synthesized under various HPHT conditions using high-resolution transmission electron microscopy (HRTEM). We find that the minimum value of $\lambda$ at $\sim 20 \mathrm{GPa}$ is related to a change in the subsequent deformation mechanism inside the preformed transformation twins from glide-set dislocations, which can slide on the $\{111\}$ planes and thus produce deformation twin lamellae, to shuffle-set ones, which cannot produce finer twin lamellae. We use the kinetic dislocation theory and density-functional-based tight-binding method to confirm the change in the deformation twinning mechanism at a critical shear stress, which accounts for the variations of $\lambda$ and $H_{\mathrm{V}}$ with the synthesis pressure. Our results indicate that twin formation mechanisms play an important role in manipulating $\lambda$ and may be employed to further enhance the properties of nt-diamond.

\section{EXPERIMENTAL SECTION}

\section{Synthesis and characterization of nt-diamond}

The synthesis of nanotwinned diamond was reported in our previous work [10]. Briefly, onion carbon nanoparticles $(20-50 \mathrm{~nm}$ in diameter, as shown in Fig. S2a, b, SI) produced from black carbon powders using an impinging streams technique were treated at pressures of 18-25 GPa and temperatures of $1850-2000^{\circ} \mathrm{C}$. The recovered samples were transparent and about $1-2 \mathrm{~mm}$ in diameter and $0.2-0.5 \mathrm{~mm}$ in height. All the samples were pure diamond 
with grain sizes of $30-50 \mathrm{~nm}$, as confirmed by the X-ray diffraction (XRD, $\mathrm{Cu} \mathrm{Ka}, \mathrm{D} 8$ Discover) patterns and TEM (Fig. S2c, d). A microhardness tester (KB 5 BVZ) was used to measure $H_{\mathrm{V}}$. The microstructural features were examined by TEM and HRTEM (JEM-2010) operating at $200 \mathrm{kV}$. The average twin thickness was derived from that of hundreds of nanotwins with the help of HRTEM measurements. The maximum temperature rise due to electron beam heating when TEM is performed at $200 \mathrm{kV}$ which was estimated to be about $2.58^{\circ} \mathrm{C}$, and the minimum incident energy for the defect formation in diamond was estimated to be $220 \mathrm{keV}$ in the [110] directions [41-43]. Thus, the electron beam effects were negligible.

\section{RESULTS AND DISCUSSION}

HRTEM measurements indicate different twin thickness distributions in five nt-diamond samples synthesized at 18-25 GPa and $1850-2000^{\circ} \mathrm{C}$ (Fig. S3, SI). Table 1 summarizes the measured Vickers hardness $H_{\mathrm{V}}$ and twin thickness $\lambda$, which reach a minimum of $\sim 5 \mathrm{~nm}$ and a maximum of $\sim 203 \mathrm{GPa}$, respectively, at $\sim 20 \mathrm{GPa}$. The variations of $\lambda$ and the corresponding XRD pattern were reported previously [10]. Samples recovered from $18 \mathrm{GPa} / 1850^{\circ} \mathrm{C}$ and $20 \mathrm{GPa} / 1850^{\circ} \mathrm{C}$ show a shoulder on the left side of the (111) peak, which exhibits a larger full width at half-maximum than samples recovered from $20 \mathrm{GPa} / 2000^{\circ} \mathrm{C}, 25$ $\mathrm{GPa} / 1850^{\circ} \mathrm{C}$, and $25 \mathrm{GPa} / 2000^{\circ} \mathrm{C}$. The shoulder was also observed in diamond synthesized by shock-wave compression and attributed to abundant stacking faults in the sample [44]. Our TEM investigations found no significant difference in the grain size distributions of the five HPHT samples, where the size of the cubic diamond grains are all in the range of $30-50 \mathrm{~nm}$, similar to that of the onion carbon precursors. Therefore, the observed shoulder and broadening of the XRD peaks for nt-diamond should arise mainly from the large number of stacking faults. Such highly dense stacking faults are rarely reported in the HPHT-synthesized diamonds but are frequently observed in heavily deformed diamonds [44]. Lonsdaleite [45], known as "hexagonal diamond," also exhibits a similar shoulder [40]. Recent HRTEM observations suggest that lonsdaleite may in fact be cubic diamond with a high concentration of twins and stacking faults [46], and the high density of stacking faults in lonsdaleite was attributed to plastic deformation.

Fig. 1a displays a typical HRTEM image of an nt-diamond synthesized at $20 \mathrm{GPa} / 1850^{\circ} \mathrm{C}$, which shows some nanotwins terminated inside a crystal. Fig. $1 \mathrm{~b}$ shows an HRTEM image of a nanotwin domain bounded by two coherent planar twin boundaries lying in the (111) plane. The planar, coherent twin boundaries suggest that the twin domain likely originated in transformation or growth. In addition, some stacking faults are observed, which are likely induced by plastic deformation through sliding of Shockley partial dislocations in the $\{111\}$ planes [36]. Fig. $1 \mathrm{c}$, on the other hand, displays an HRTEM image and the corresponding fast Fourier transform (FFT) pattern of some complex twins. Two parallel twin boundaries, with a separation distance of less than $4 \mathrm{~nm}$, can be seen to terminate inside a larger nanotwin domain. Such terminated twin boundaries can be formed only through the motions of Shockley partial dislocations emitted from the grain boundary (beyond the right marge of this figure) during plastic deformation [47]. In other words, the narrow twin domain bounded by two parallel boundaries is a deformation twin produced after the nanodiamond formed. Fig. $1 \mathrm{~d}$ is an enlargement of the area containing the lower-right twin boundary (blue box) in Fig. 1c. On the basis of the stacking sequence of (111) planes on both sides of the twin boundary, one can easily distinguish whether the twin plane is a glide or a shuffle plane. For example, in Fig. 1d, the stacking sequence across the twin plane is symmetrical (... CBABC …), so the twin plane must be a shuffle plane. Glide-set sliding, on the other hand, can be identified in the enlarged HRTEM image shown in Fig. 1e, which is adjacent to the terminated twin boundaries (the red box

Table 1 Vickers hardness $H_{\mathrm{V}}$ and average twin thickness $\lambda$ of the nt-diamond samples synthesized at $18 \mathrm{GPa} / 1850^{\circ} \mathrm{C}, 20 \mathrm{GPa} / 1850^{\circ} \mathrm{C}, 20 \mathrm{GPa} / 2000^{\circ} \mathrm{C}$, and $25 \mathrm{GPa} / 2000^{\circ} \mathrm{C}$

\begin{tabular}{cccc}
\hline Samples & $P(\mathrm{GPa}) / T\left({ }^{\circ} \mathrm{C}\right)$ & $H_{\mathrm{V}} \pm$ s.d. $(\mathrm{GPa})$ & Twin thickness \pm s.d. $(\mathrm{nm})$ \\
\hline O363 & $18 / 1850$ & $191.8 \pm 9.3$ & $5.4 \pm 0.4$ \\
S379 & $18 / 1850$ & $191.7 \pm 9.3$ & $5.2 \pm 0.4$ \\
P368 & $20 / 1850$ & $198.7 \pm 32.5$ & $5.0 \pm 0.2$ \\
V376 & $20 / 2000$ & $203.6 \pm 12.0$ & $6.2 \pm 0.8$ \\
W377 & $25 / 2000$ & $193.6 \pm 21.7$ & $7.4 \pm 0.2$ \\
M363 & $25 / 2000$ & $175.4 \pm 18.2$ & \\
\hline
\end{tabular}




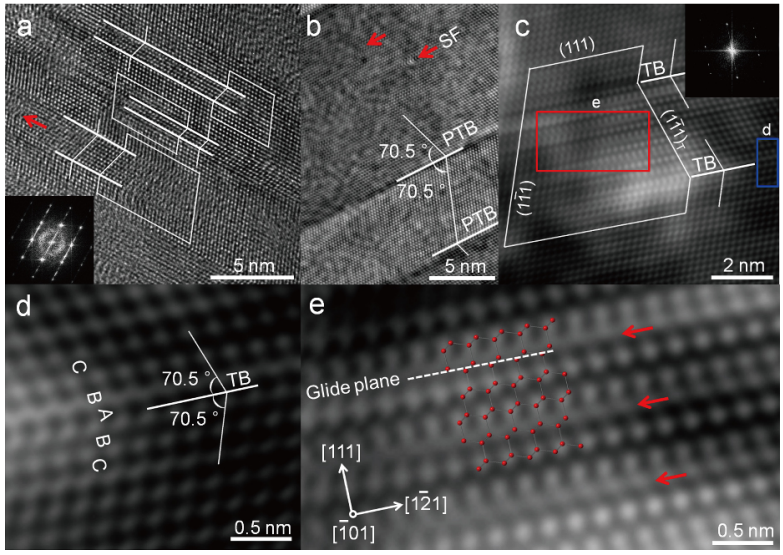

Figure 1 TEM observations of an nt-diamond sample synthesized at $20 \mathrm{GPa} / 1850^{\circ} \mathrm{C}$. (a) Raw HRTEM image of a nanograin in nt-diamond, showing three nanotwins terminated inside a nanograin. A stacking fault is marked with a red arrow. The white polygons indicate three sets of (111) planes. (b) HRTEM image of the typical phase transformation twins viewed along the [101] zone axis of diamond. Several stacking faults (SFs) and phase transformation twin boundaries (PTBs) are marked. (c) HRTEM image and the corresponding FFT pattern (inset) of the deformation twins. Two terminated twin boundaries (TBs) are labeled. The white polygon indicates three sets of (111) planes. On the right-hand side of the polygon, the zigzag line shows the relation between (111)and its twin $(1 \overline{1} 1)_{\mathrm{T}}$, where the deformation twins end inside a phase transformation twin. (d) Enlarged HRTEM image (area corresponding to the blue box in c) showing a deformation twin boundary, with a symmetrical stacking sequence of (111) planes, as labeled. (e) Enlarged HRTEM image (area corresponding to the red box in c) superimposed with a structural model showing the atomic positions. Three glide planes are marked with red arrows.

within the white polygon in Fig. 1c). The nt-diamond sample synthesized at $18 \mathrm{GPa} / 1850^{\circ} \mathrm{C}$ exhibits microstructural features similar to those described above, as shown in Fig. S4 (SI). These observations show that the twin thickness of nt-diamond can be further reduced by subsequent deformation twinning through glide-set partial dislocations inside the pre-existing transformation or growth twins.

Fig. 2a-c shows typical bright-field TEM images of nt-diamonds synthesized at $20 \mathrm{GPa} / 2000^{\circ} \mathrm{C}, 25 \mathrm{GPa} / 1850^{\circ} \mathrm{C}$, and $25 \mathrm{GPa} / 2000^{\circ} \mathrm{C}$, respectively. Clearly, the $20 \mathrm{GPa}$ sample contains a higher density of nanotwins with smaller $\lambda$ than those synthesized at $25 \mathrm{GPa}$. In fact, more twin lamellae with finer thickness $(<6 \mathrm{~nm})$ can be found in the $20 \mathrm{GPa}$ sample. On the basis of the observations reported in Fig. 1, we interpret these fine lamellae as deformation twins formed by glide-set dislocations after the formation of transformation or growth twins. The enlarged HRTEM image from the selected area in Fig. $2 \mathrm{~b}$ (the $25 \mathrm{GPa} / 1850^{\circ} \mathrm{C}$ sample) and the corresponding FFT pattern are displayed in Fig. 2d. Multiple parallel twins are present without

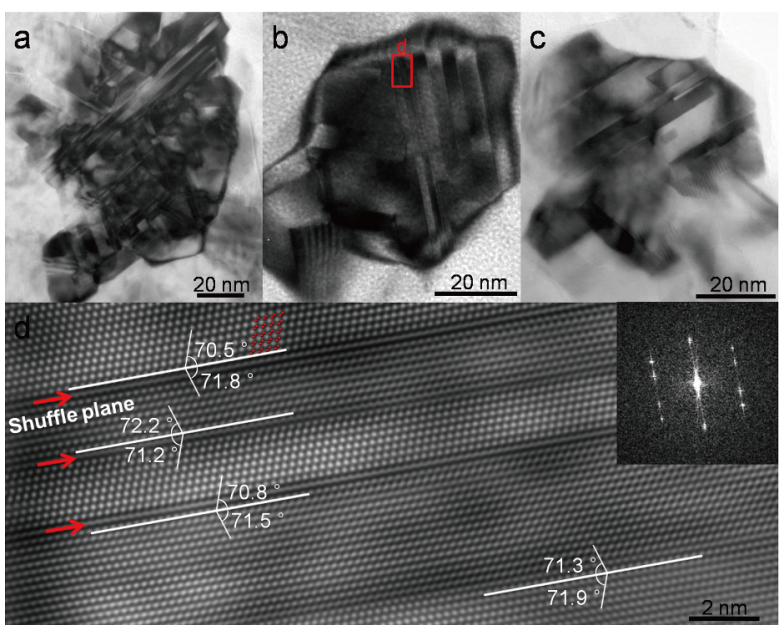

Figure 2 TEM observations of three nt-diamond samples synthesized under different conditions. (a-c) Bright-field TEM images of nanograins in three nt-diamond samples recovered from $20 \mathrm{GPa} / 2000^{\circ} \mathrm{C}$, $25 \mathrm{GPa} / 1850^{\circ} \mathrm{C}$, and $25 \mathrm{GPa} / 2000^{\circ} \mathrm{C}$, respectively. (d) HRTEM image (corresponding to the red box in b) viewed along the [101] zone axis and associated FFT pattern (inset). Three sets of twin boundaries and twinning angles are labeled. The shuffle planes are recognized on the basis of the structure model and marked with red arrows.

stacking faults. Note that the measured twinning angles depart slightly from the ideal value of $70.53^{\circ}$. When a perfect dislocation slide parallels to the (111) twin boundary and completely passes through the twin domain, it leaves no imprint that can be observed by HRTEM. However, if the sliding of these perfect dislocations terminates within a twin, incommensurate shifts of atoms on the adjacent (111) planes, thereby inducing slight variations in the twinning angles. Therefore, the deviation of the twin angles from $70.53^{\circ}$ indicates the presence of shuffle-set dislocations. Our HRTEM observations indicate that deformation by shuffle-set dislocations dominates in the two nt-diamond samples synthesized at $25 \mathrm{GPa}$, as shown in Fig. 2. However, such shuffle-set deformation inside transformation or growth nanotwins cannot further reduce the twin thickness.

The above TEM observations provide strong experimental evidence that the mechanism of plastic deformation in pre-existing transformation and growth nanotwins changes from being controlled by glide-set dislocation to being controlled by shuffle-set dislocation at a critical pressure between 20 and $25 \mathrm{GPa}$. Below the threshold pressure, glide-set sliding dominates deformation, resulting in dense stacking faults and further reduced twin thickness. Above the threshold, shuffle-set sliding dominates, and no stacking fault or finer twin can be produced. 
We have shown above that a significant proportion of the nanotwins originate in deformation. Previous molecular dynamics simulations and experimental observations [48] indicated that dislocation nucleation at grain boundary-twin intersections governs plastic deformation of nanotwinned materials, unlike the case in coarser-grained materials, where dislocations multiply within the grains. These intersections tend to have the highest stress concentration and are the sources of Shockley partial dislocations, which propagate in the $\{111\}$ planes and produce additional, finer deformation twin lamellae [48]. In this case, the dislocation motion is no longer limited by the nanoscale crystallites in nanotwinned materials.

The situation in diamond is more complex because diamond has two dislocation slip modes in the $\{111\}$ planes, namely, glide-set and shuffle-set dislocations (Fig. S1, SI). To evaluate the competing plastic deformation mechanisms of glide-set and shuffle-set dislocations within transformation or growth nanotwins, we performed theoretical investigations using kinetic dislocation theory [49] combined with the density-functional-based tight-binding method [50]. The strain rate $\varepsilon$ of a deforming nt-diamond grain facilitated by the two dislocation sets can be used as a criterion to determine which slip mode governs plastic deformation of pre-existing twins under given stress and temperature conditions. Specifically, the slip mode inducing higher $\bar{\varepsilon}$ will dominate the plastic deformation. The value of $\bar{\varepsilon}$ induced by the two slip modes at an applied shear stress $(\tau)$ and temperature $(T)$ can be estimated as [48]

$$
\bar{\varepsilon}=\frac{d \nu_{\mathrm{D}}}{\lambda \exp \left(\frac{\Delta U}{\kappa T}-\frac{S V^{*}}{\kappa T} \tau\right)},
$$

where $d, v_{\mathrm{D}}$, and $k$ are the grain size, Debye frequency, and Boltzmann constant, respectively. $\Delta U$ is the activation energy at $0 \mathrm{~K}, S$ is a factor representing the local stress concentration and geometry, and $V^{*}$ is the activation volume. We assume, somewhat arbitrarily, that the two types of dislocations are immobile when the strain rate is less than $10^{-6}$ $\mathrm{s}^{-1}$. Above $10^{-6} \mathrm{~s}^{-1}$, the slip mode with the higher strain rate is the dominant deformation mechanism. Details of the determination of all the parameters used in Equation (1) are given in the Supporting information, and the values are listed in Table S1 (SI).

A dislocation mobility map of nt-diamond (Fig. 3) is constructed using the $\bar{\varepsilon}$ values of the two slip modes calculated at different $\tau$ and $T$ values. Plastic deformation cannot

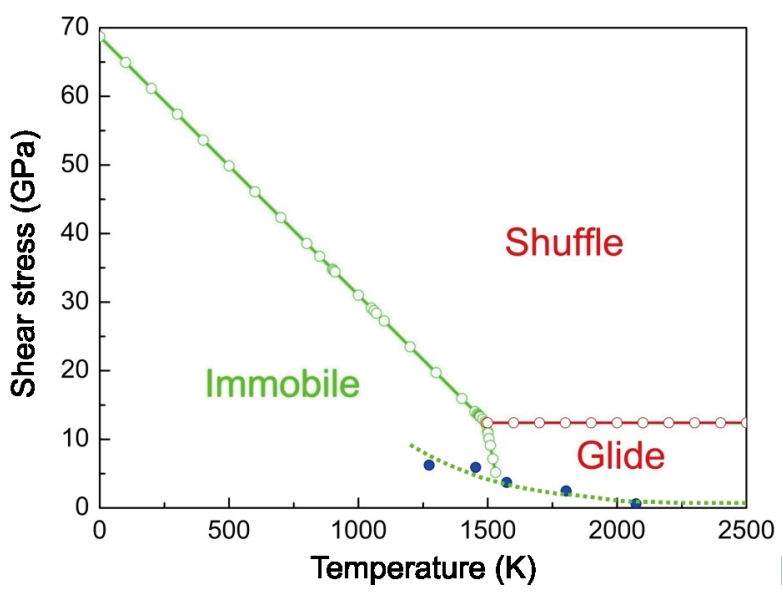

Figure 3 Dislocation mobility map of nt-diamond constructed using $\bar{\varepsilon}$ values calculated at different $\tau$ and $T$ values. The "Immobile" zone corresponds to calculated strain rates below $10^{-6} \mathrm{~s}^{-1}$. In the temperature range corresponding to the synthesis experiments, plastic deformation of diamond nanotwins is dominated by glide-set dislocations at lower shear stresses and by shuffle-set dislocations at higher shear stresses. In this map, the yield strength of diamond at $\sim 1273, \sim 1453,1573,1803$, and $2073 \mathrm{~K}$, which is derived from reference [20], is also labeled (blue points). The values are estimated according to the empirical relation $H=$ $C \sigma$, where $C=2.5$.

be initialized at low temperature and low shear stress (the zone labeled "Immobile" in Fig. 3, where the strain rates are below $10^{-6} \mathrm{~s}^{-1}$ ). In the temperature range of nt-diamond syntheses, glide-set dislocations first govern the plastic deformation ("Glide" zone in Fig. 3) with increasing $\tau$. When $\tau$ exceeds a critical value, shuffle-set dislocations then become dominant ("Shuffle" zone in Fig. 3). It should be noted that our model is not suitable for estimating the yield strength of diamond above $1500 \mathrm{~K}$; thus, a green dashed line is plotted according to the yield strength of diamond in reference [20] and placed below the glide zone as a token boundary between the immobile and glide zones.

This analysis is not intended to provide a quantitative evaluation of the competing mechanisms, as very little is known about plastic deformation of nanodiamonds, especially at elevated temperatures. The first reported roomtemperature stress-strain curves for nanodiamonds were obtained for diamond pillars with diameters of $0.2-1 \mu \mathrm{m}$ inside a scanning electron microscope [51], and the results suggested that diamonds below $\sim 10 \mathrm{~nm}$ in diameter would approach the ideal shear strength of $G / 2 \pi$, or $\sim 80 \mathrm{GPa}$, where $G$ is the shear modulus of diamond. This provides a rough checkpoint for the validity of our analysis. Indeed, the critical shear stress for dislocation motion at $0 \mathrm{~K}$, corresponding to the point on the boundary between the immobile and shuffle zones at $0 \mathrm{~K}$, reaches $\sim 70 \mathrm{GPa}$, as shown 
in Fig. 3.

X-ray peak broadening analysis [20] shows that the yield strength of diamond powders with a grain size of $\sim 5 \mu \mathrm{m}$ decreases from $\sim 15 \mathrm{GPa}$ at $1300 \mathrm{~K}$ to $\sim 1.5 \mathrm{GPa}$ at $2100 \mathrm{~K}$. Although no high-temperature shear strength data are available for nanodiamonds, it appears safe to assume that their shear strengths are generally below $\sim 5 \mathrm{GPa}$ at temperatures above $1500 \mathrm{~K}$. In the two dislocation mobile zones (i.e., the glide and shuffle zones), our results show that the dominant deformation mechanism is determined primarily by $\tau$. During the synthesis experiments, the shear stresses are produced by grain contacts due to elastic anisotropy of the diamond crystals. At a given temperature, the shear stress increases with increasing pressure. This explains why the $25 \mathrm{GPa}$ samples contain mostly shuffle dislocations: the higher shear stress in these samples may exceed the shearing threshold (Fig. 3). In contrast, the stress in the $20 \mathrm{GPa}$ samples is below the threshold, so the glide-set dislocations dominate. The operation of the deformation mechanisms in nt-diamond differs from that in micron-sized diamonds, which also depends on the synthesis temperature [36].

\section{CONCLUSIONS}

In conclusion, the observed turnover in nanotwin thickness as a function of pressure is due to the combined contribution of two processes during the synthesis of nt-diamond. The first process is transformation and growth of diamond from onion carbon precursors. At similar synthesis temperatures, the nucleation rate is controlled primarily by pressure. The thickness of the nanotwins formed during this process decreases monotonically with increasing synthesis pressure. The second process is mechanical twinning due to plastic deformation, which may further reduce $\lambda$. At our synthesis temperatures (all below $50 \%$ of the melting temperature of diamond), the amplitude of the deviatoric stress is expected to increase with synthesis pressure owing to the increased elastic anisotropy of diamond. Once the deviatoric stress level exceeds a certain threshold, twin-producing glide-set dislocations cease to be the dominant deformation mechanism, and $\lambda$ cannot be further reduced. This threshold stress appears to be reached at a synthesis pressure between 20 and $25 \mathrm{GPa}$. According to this analysis, the properties of nt-diamond may be further enhanced by optimizing the synthesis conditions favoring the glide-set mechanism and further refinement of $\lambda$.

Received 26 November 2016; accepted 26 December 2016; published online 16 January 2017

1 Yan C, Mao H, Li W, et al. Ultrahard diamond single crystals from chemical vapor deposition. Phys Status Solidi A, 2004, 201:
R25-R27

2 Sumiya H, Harano K. Distinctive mechanical properties of nanopolycrystalline diamond synthesized by direct conversion sintering under HPHT. Diamond Related Mater, 2012, 24: 44-48

3 Irifune T, Kurio A, Sakamoto S, et al. Materials: ultrahard polycrystalline diamond from graphite. Nature, 2003, 421: 599-600

4 Sumiya H, Irifune T. Hardness and deformation microstructures of nano-polycrystalline diamonds synthesized from various carbons under high pressure and high temperature. J Mater Res, 2007, 22: 2345-2351

5 Hall EO. The deformation and ageing of mild steel: III discussion of results. Proc Phys Soc B, 1951, 64: 747-753

6 Petch NJ. The cleavage strength of polycrystals. J Iron Steel Ins, 1953, 174: $25-28$

7 Veprek S. Recent search for new superhard materials: go nano! J Vacuum Sci Tech A-Vacuum Surfaces Films, 2013, 31: 050822

8 Lu L, Chen X, Huang X, et al. Revealing the maximum strength in nanotwinned copper. Science, 2009, 323: 607-610

9 Tian Y, Xu B, Yu D, et al. Ultrahard nanotwinned cubic boron nitride. Nature, 2013, 493: 385-388

10 Huang Q, Yu D, Xu B, et al. Nanotwinned diamond with unprecedented hardness and stability. Nature, 2014, 510: 250-253

11 Gao F, He J, Wu E, et al. Hardness of covalent crystals. Phys Rev Lett, 2003, 91: 015502

12 Guo X, Li L, Liu Z, et al. Hardness of covalent compounds: roles of metallic component and d valence electrons. J Appl Phys, 2008, 104: 023503

13 Tian Y, Xu B, Zhao Z. Microscopic theory of hardness and design of novel superhard crystals. Int J Refractory Met Hard Mater, 2012, 33: $93-106$

14 Xu B, Tian Y. Ultrahardness: measurement and enhancement. J Phys Chem C, 2015, 119: 5633-5638

$15 \mathrm{Xu} B$, Tian Y. Superhard materials: recent research progress and prospects. Sci China Mater, 2015, 58: 132-142

16 Zhao Z, Xu B, Tian Y. Recent advances in superhard materials. Annu Rev Mater Res, 2016, 46: 383-406

17 Brookes CA. Plastic deformation and anisotropy in the hardness of diamond. Nature, 1970, 228: 660-661

18 Humble P, Hannink RHJ. Plastic deformation of diamond at room temperature. Nature, 1978, 273: 37-39

19 Mao HK, Bell PM. High-pressure physics: sustained static generation of 1.36 to 1.72 megabars. Science, 1978, 200: 1145-1147

20 Weidner DJ, Wang Y, Vaughan MT. Strength of diamond. Science, 1994, 266: 419-422

21 Blumenau AT, Heggie MI, Fall CJ, et al. Dislocations in diamond: core structures and energies. Phys Rev B, 2002, 65: 205205

22 Mussi A, Eyidi D, Shiryaev A, et al. TEM observations of dislocations in plastically deformed diamond. Phys Status Solidi A, 2013, 210: 191-194

23 Pizzagalli L, Demenet JL, Rabier J. Theoretical study of pressure effect on the dislocation core properties in semiconductors. Phys Rev B, 2009, 79: 045203

24 Saka H, Yamamoto K, Arai S, et al. In-situ TEM observation of transformation of dislocations from shuffle to glide sets in Si under supersaturation of interstitials. Philos Mag, 2006, 86: 4841-4850

25 Li Z, Picu RC. Shuffle-glide dislocation transformation in Si. J Appl Phys, 2013, 113: 083519-083519

26 Gaillou E, Post JE, Bassim ND, et al. Spectroscopic and microscopic characterizations of color lamellae in natural pink diamonds. Diamond Related Mater, 2010, 19: 1207-1220

27 Blumenau AT, Jones R, Frauenheim T, et al. Dislocations in diamond: dissociation into partials and their glide motion. Phys Rev 


\section{B, 2003, 68: 014115}

28 Duesbery MS. Dislocation motion in silicon: the shuffle-glide controversy. Philos Mag Lett, 1996, 74: 253-258

29 Yacoot A, Moore M, Machado WG. Twinning in natural diamond. I. Contact twins. J Appl Crystallogr, 1998, 31: 767-776

30 Machado WG, Moore M, Yacoot A. Twinning in natural diamond. II. Interpenetrant cubes. J Appl Crystallogr, 1998, 31: 777-782

31 Hidetaka S, Hideki I. Atomic structure of fivefold twin center in diamond film. Diam Relat Mater, 2005, 14: 109-112

32 Delclos S, Dorignac D, Phillipp F, et al. Ultra-high resolution electron microscopy investigation of growth defects in CVD diamond films: twin interactions and fivefold twin centres. Diamond Related Mater, 2000, 9: 346-350

33 Tomlinson EL, Howell D, Jones AP, et al. Characteristics of HPHT diamond grown at sub-lithosphere conditions (10-20GPa). Diamond Related Mater, 2011, 20: 11-17

34 Kulnitskiy B, Perezhogin I, Dubitsky G, et al. Polytypes and twins in the diamond-lonsdaleite system formed by high-pressure and high-temperature treatment of graphite. Acta Crystallogr B Struct Sci Cryst Eng Mater, 2013, 69: 474-479

35 Tanigaki K, Ogi H, Sumiya H, et al. Observation of higher stiffness in nanopolycrystal diamond than monocrystal diamond. Nat Commun, 2013, 4: 2343

36 Britun VF, Oleynik GS, Semenenko NP. Deformation processes during high-pressure sintering of the diamond powders produced by catalytic synthesis. J Mater Sci, 1992, 27: 4472-4476

37 Walmsley JC, Lang AR. Transmission electron microscopic observations of deformation and microtwinning in a synthetic diamond compact. J Mater Sci Lett, 1983, 2: 785-788

38 Titkov SV, Krivovichev SV, Organova NI. Plastic deformation of natural diamonds by twinning: evidence from X-ray diffraction studies. Mineral Mag, 2012, 76: 143-149

39 Howell D, Piazolo S, Dobson DP, et al. Quantitative characterization of plastic deformation of single diamond crystals: a high pressure high temperature (HPHT) experimental deformation study combined with electron backscatter diffraction (EBSD). Diamond Related Mater, 2012, 30: 20-30

40 Churchman AT, Geach GA, Winton J. Deformation twinning in materials of the A4 (diamond) crystal structure. Proc R Soc Lond A, 1956, 238: 194-205

41 Dong H, Wen B, Melnik R. Relative importance of grain boundaries and size effects in thermal conductivity of nanocrystalline materials. Sci Rep, 2014, 4: 7037

42 Koike J, Parkin DM, Mitchell TE. Displacement threshold energy for type IIa diamond. Appl Phys Lett, 1992, 60: 1450-1452

43 Wang L, Zheng K, Zhang Z, et al. Direct atomic-scale imaging about the mechanisms of ultralarge bent straining in Si nanowires.
Nano Lett, 2011, 11: 2382-2385

44 Shames AI, Mogilyansky D, Panich AM, et al. XRD, NMR, and EPR study of polycrystalline micro- and nano-diamonds prepared by a shock wave compression method. Phys Status Solidi A, 2015, 212: 2400-2409

45 Frondel C, Marvin UB. Lonsdaleite, a hexagonal polymorph of diamond. Nature, 1967, 214: 587-589

46 Nemeth P, Garvies LAJ, Aoki T, et al. Lonsdaleite is faulted and twinned cubic diamond and does not exist as a discrete material. Nat Commun, 2014, 5: 5447

47 Zhu YT, Liao XZ, Wu XL. Deformation twinning in nanocrystalline materials. Prog Mater Sci, 2012, 57: 1-62

48 Li X, Wei Y, Lu L, et al. Dislocation nucleation governed softening and maximum strength in nano-twinned metals. Nature, 2010, 464: $877-880$

49 Thomson RM, Balluffi RW. Kinetic theory of dislocation climb. I. General models for edge and screw dislocations. J Appl Phys, 1962, 33: $803-816$

50 Porezag D, Frauenheim T, Köhler T, et al. Construction of tightbinding-like potentials on the basis of density-functional theory: application to carbon. Phys Rev B, 1995, 51: 12947-12957

51 Wheeler JM, Raghavan R, Wehrs J, et al. Approaching the limits of strength: measuring the uniaxial compressive strength of diamond at small scales. Nano Lett, 2016, 16: 812-816

Acknowledgments This work was supported by the National Natural Science Foundation of China (51421091, 51332005, 51572225, 51272227, 51172197,51525205 and 51672239) and the US National Science Foundation (EAR-1361276).

Author contributions Tian Y conceived the project. Huang Q synthesized the samples. $\mathrm{Hu} \mathrm{W}$ performed the TEM and HRTEM measurements. Wen B and Xiao J performed the theoretical calculations. Tian Y, Xu B, and $\mathrm{Hu}$ W wrote the paper. All authors contributed to the general discussion.

Conflict of interest The authors declare that they have no conflict of interest.

Supplementary information The glide and shuffle planes in diamond (Fig. S1); TEM and HRTEM images of the precursor, and TEM image and selected-area electron diffraction pattern of the nt-diamond (Fig. S2); twin thickness distributions of nt-diamonds synthesized under different conditions (Fig. S3); TEM observations of an nt-diamond sample synthesized at $18 \mathrm{GPa} / 1850^{\circ} \mathrm{C}$ (Fig. S4); methods of determining the parameters in Equation (1) for glide-set and shuffle-set slipping deformation mechanisms and the calculation parameters used in Equation (1) for glide-set and shuffle-set slipping deformation mechanisms (Table S1) are available in the online version of this paper. 

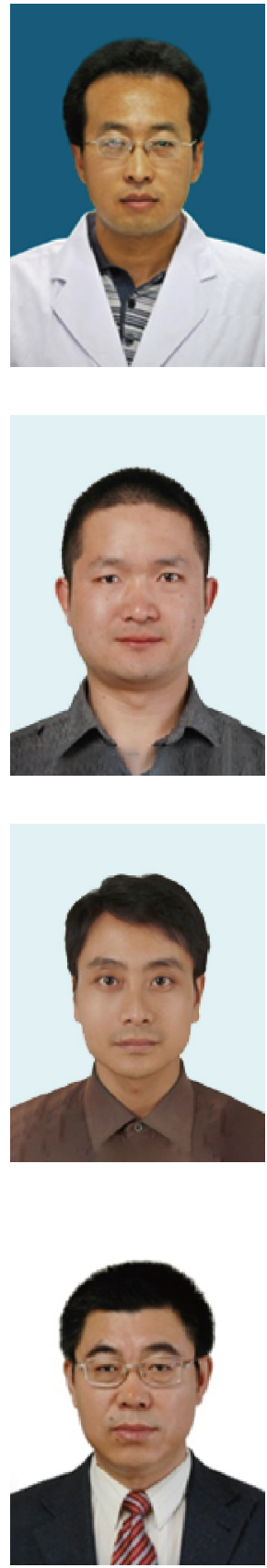

Wentao Hu is a senior experimentalist at the College of Materials Science and Engineering, Yanshan University. He received his $\mathrm{PhD}$ degree from Yanshan University in 2013. His research interests include superhard materials and transmission electron microscopy.
Quan Huang works at HPSTAR as a postdoctoral fellow. He received his PhD degree from Yanshan University in 2015. His research interests include the design and synthesis of novel metastable materials under high pressure and high temperature.
Bin Wen is a professor at the College of Materials Science and Engineering, Yanshan University. He received his PhD degree from Dalian University of Technology in 2006. His research interests include theoretical calculation and molecular dynamics simulation.

\section{塑性变形在纳米孪晶金刚石微观组织超细化中的作用}

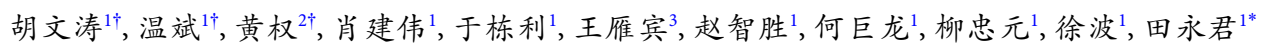

摘要 在高温高压条件下以洋葱碳为原料合成的纳米孪晶金刚石具有前所未有的硬度和稳定性, 且二者随纳米孪晶厚度的减小而提高. 目 前为止, 在金刚石纳米晶中纳米孪晶的形成机制尚不明确. 本研究通过分析在不同条件下合成的纳米孪晶金刚石块材中的孪晶厚度, 发现 在合成压力约为 $20 \mathrm{GPa}$ 时孪晶厚度达到一个极小值 $(5 \mathrm{~nm})$. TEM结果表明在合成压力低于 $20 \mathrm{GPa}$ 时, 纳米孪晶金刚石中同时存在因马氏 体相变而形成的相变孪晶和塑性形变所导致的形变孪晶. 针对马氏体相变后形成的相变孪晶内部塑性变形, 基于密度泛函理论的紧束缚 方法和位错运动学理论的分析表明: 纳米孪晶金刚石的塑性变形存在一个依赖于压力的机制转变, 而机制转变的临界压力能够解释孪晶 厚度随压力变化时出现的极小值. 本研究对通过优化合成条件制备出更高硬度和稳定性的纳米孪晶金刚石具有指导意义. 